\title{
Nouveaux échafaudages protéiques modulaires pour les récepteurs couplés aux protéines $G$
}

Les récepteurs couplés aux protéines G (RCPG) représentent la plus grande famille de récepteurs membranaires impliqués dans la transmission intracellulaire du signal. Les travaux réalisés au cours des 20 dernières années ont permis de proposer un modèle général permettant d'expliquer leur mode d'action. Selon ce modèle, le complexe de signalisation se compose de trois protéines: un récepteur monomérique, une protéine $\mathrm{G}$ hétéro-trimérique $(G \alpha \beta \gamma)$ et un effecteur qui peut être une enzyme, un canal ionique ou un transporteur. En entraînant l'échange du GDP lié à la sous-unité $\alpha$ de la protéine $\mathrm{G}$ pour une molécule de GTP, l'activation du récepteur favorise la dissociation de $\mathrm{G} \alpha$ du dimère G $\beta \gamma$. Ces sous-unités une fois dissociées interagissent avec les effecteurs qui leur sont propres pour en moduler l'activité. Les seconds messagers et les mouvements ioniques ainsi produits induisent ensuite les changements d'activité cellulaire sous-tendant les réponses biologiques. La beauté et la simplicité relative de ce modèle en ont fait une vérité presque dogmatique qui implique une cascade d'interactions transitoires entre des protéines qui sont mobiles dans le plan de la membrane cellulaire. Toutefois, des résultats récents remettent en question plusieurs aspects de ce modèle. En particulier, des interactions protéine-protéine plus nombreuses que celles anticipées suggèrent l'échafaudage de complexes modulaires de signalisation qui seraient localisés dans des compartiments spécialisés de la membrane plasmique.

Dans un article récemment publié dans la revue Science [1], les groupes de Caron et Lefkowitz à l'université Duke (Durham, NC, USA) rapportent que la protéine $\beta$-arrestine-1, connue jusqu'à présent pour son rôle dans la désensibilisation des récepteurs [2], est au centre d'un tel échafaudage protéique qui serait impliqué dans l'activation de la voie des MAP kinases.

\section{La double personnalité de la $\beta$-arrestine}

La $\beta$-arrestine-1 [3] est un des membres de la famille des arrestines, découvertes pour leur capacité d'interagir avec les RCPG sous leur forme phosphorylée par des protéine-kinases spécifiques, les kinases des RCPG ou GRK. Cette interaction de forte affinité entre l'arrestine et le récepteur phosphorylé est responsable du découplage fonctionnel du récepteur de la protéine $\mathrm{G}$ et donc de la désensibilisation de la voie de signalisation [2]. De plus, grâce à des domaines d'interactions avec la chaîne lourde [4] et les protéines adaptatrices de la clathrine [5], les arrestines entraînent les récepteurs vers les puits tapissés de clathrines conduisant à leur endocytose. Jusqu'à tout récemment, cette séquence d'événements était considérée strictement comme un mécanisme d'inactivation permettant d'atténuer la réponse à une stimulation soutenue.

Par ailleurs, on a récemment montré que plusieurs RCPG peuvent activer les différentes cascades de signalisation des MAP kinases, indépendamment de l'activation des effecteurs classiques des RCPG tels que l'adénylyl cyclase et la phospholipase C. L'activation de ces voies mitogé- niques nécessite la phosphorylation sur des tyrosines de protéines comme Shc, Gab-1 et Grb-2 et l'assemblage de celles-ci en complexes protéiques menant à l'activation de Ras [6]. Plusieurs résultats suggèrent aussi un rôle pour les sous-unités $\beta \gamma$ des protéines $\mathrm{G}$ et pour la tyrosine kinase cytoplasmique Src dans cette voie d'activation [7]. Toutefois, le lien entre l'activation des RCPG et l'activation de Src demeurait mal compris. Les résultats obtenus par l'équipe de Duke permettent maintenant d'établir un lien entre les RCPG et l'activation de Src. En effet, les chercheurs ont pu démontrer par des approches d'immunofluorescence et de coimmunoprécipitation que la liaison, dépendante de l'agoniste, de la $\beta$ arrestine au récepteur $\beta 2$-adrénergique, conduit à la formation d'un complexe multiprotéique comprenant le récepteur, la $\beta$-arrestine-1 et cSrc. Le recrutement de c-Src dans ce complexe dépend d'une interaction entre le domaine SH3 de Src et une région riche en proline dans la partie amino-terminale de la $\beta$-arrestine- 1 . L'importance fonctionnelle de cette interaction est illustrée par l'observation que divers mutants de la $\beta$-arrestine qui ne peuvent plus s'associer à Src mais peuvent toujours se lier au récepteur et à la clathrine ont des effets dominants négatifs sur l'activation des MAP kinases Erk-1 et Erk-2 par le récepteur $\beta 2$-adrénergique. Réciproquement, des mutants de la $\beta$ arrestine qui ne peuvent interagir avec la clathrine mais s'associent normalement au récepteur et à Src inhibent aussi cette voie de signalisation suggérant que non seulement le recrutement de Src mais aussi le ciblage du complexe vers les puits de 
clathrine sont nécessaires à l'activation des MAP kinases. Ainsi, la fonction de l'échafaudage protéique serait le recrutement à la membrane de la forme active de la kinase Src, le ciblage du complexe récepteur- $\beta$ arrestine-Src vers les puits de clathrine et l'activation de la cascade de signalisation conduisant à la stimulation de Ras et de là à l'activation des MAP kinases (figure 1). L'observation précédemment publiée qu'un mutant de la dynamine ayant un effet dominant négatif sur l'internalisation du récepteur $\beta 2$-adrénergique prévient aussi l'activation de Erk-1 et Erk-2 par ce récepteur [8], suggère que sont nécessaires, non seulement le ciblage du complexe vers les puits de clathrine, mais aussi l'endocytose du complexe signalétique. Un pré-requis similaire pour l'internalisation a été proposé pour l'activation de la voie des MAP kinases par le récepteur de l'EGF [9].

\section{La désensibilisation}

n'est plus ce qu'elle était...

Un des aspects fascinants de ces observations réside dans le fait que le recrutement de Src et le ciblage du complexe vers les puits de clathrine résultent d'un processus qui conduit au découplage fonctionnel et physique du récepteur de sa protéine $G$ (Gs dans le cas du récepteur $\beta 2$-adrénergique) et à la désensibilisation de la réponse de son effecteur classique (l'adénylyl cyclase dans ce cas). Ainsi, c'est un récepteur "désensibilisé » qui peut activer les MAP kinases. Cela nous force donc à considérer les événements de désensibilisation résultant de la phosphorylation des récepteurs par les GRK et de la liaison de la $\beta$-arrestine non plus comme une inactivation du récepteur mais plutôt comme une réorganisation modulaire différente de partenaires protéiques favorisant une voie de signalisation au détriment d'une autre. Dans ce contexte on comprend mieux le rôle des sous-unités $\beta \gamma$ des protéines $G$ dans l'activation des MAP kinases [10]. En effet, le recrutement à la membrane plasmique des protéines GRK-2 et GRK-3, qui est nécessaire à la phosphorylation du récepteur préalable à la liai- interaction entre les sous-unités $\beta \gamma$ [11], rendues disponibles lors de l'activation de la protéine $\mathrm{G}$, et un domaine d'homologie de la pleckstrine dans la partie carboxy-terminale des kinases [12] (figure 1).

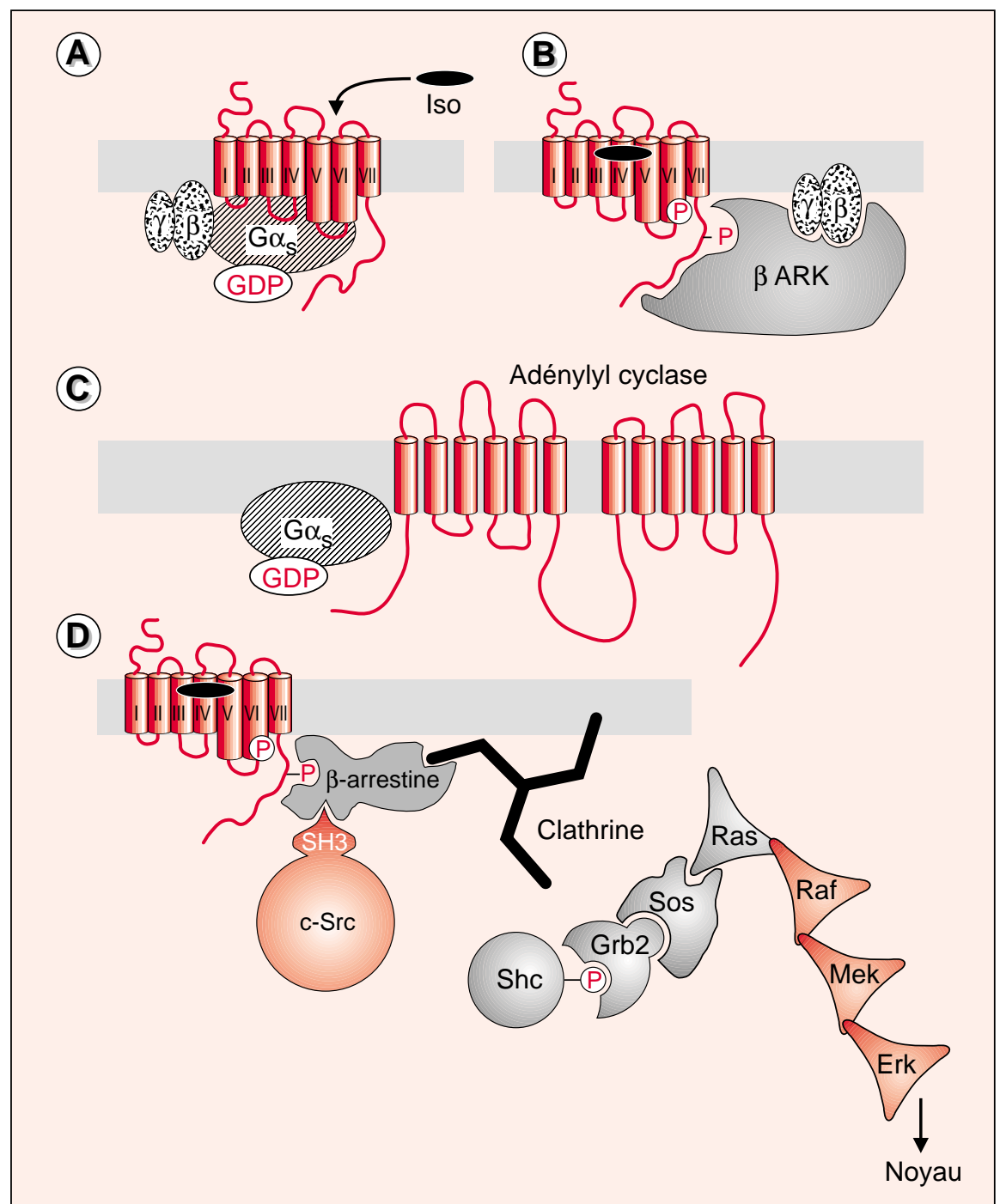

Figure 1. Rôle de la $\beta$-arrestine dans la désensibilisation de la voie de l'adénylyl cyclase et dans I'activation de la voie des MAP kinases stimulées par le récepteur $\beta 2$-adrénergique. L'activation du récepteur par un agoniste comme I'isoprotérénol (A), entraîne l'échange du GDP pour une molécule de GTP sur la sous-unité $\alpha$ de la protéine $G$, provoquant la dissociation de la sous-unité $G \alpha_{s}$ du dimère $G \beta \gamma$. G $\alpha_{\text {s }}$ liée au GTP peut ensuite interagir avec I'adénylyl

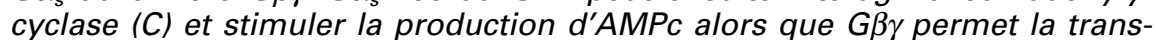
location de la GRK ( $\beta A R K)$ qui catalyse la phosphorylation du récepteur $(B)$. La présence de phosphate sur certaines sérines et thréonines de la queue cytosolique favorise la liaison de la $\beta$-arrestine au récepteur (D). Cette dernière empêche la réassociation du récepteur à $G \alpha$ entraînant une diminution dans le temps de la capacité de stimulation de l'adénylyl cyclase mais permet le recrutement de $c$-Src et de la clathrine. L'interaction du récepteur avec la clathrine est responsable de son ciblage vers les puits tapissés de clathrine alors que le recrutement et l'activation de c-Src engagent la cascade d'événements menant à l'activation de Erk. 
Une autre observation surprenante de l'étude indique que seule une partie du voile recouvrant les échafaudages de signalisation a été levée. En effet, alors que la $\beta$-arrestine cytosolique est phosphorylée, seule la forme déphosphorylée semble pouvoir interagir avec le récepteur et Src, suggérant qu'une phosphatase serait requise. Par ailleurs, l'assemblage de modules protéiques distincts semble nécessaire pour expliquer l'activation de la voie des MAP kinases par d'autres RCPG. Par exemple, bien que les récepteurs $\beta 3$-adrénergique [13] et AT1 de l'angiotensine [14] puissent stimuler Erk-1 et Erk-2, le premier n'est pas un substrat pour les GRK et n'est pas acheminé vers les puits tapissés de clathrine en réponse à une stimulation $[15,16]$ et le second est internalisé de façon indépendante de la $\beta$-arrestine [17]. En accord avec cette dernière observation, les mutants de la $\beta$-arrestine qui ne peuvent lier Src ou la clathrine sont sans effet sur la stimulation de Erk-1 et Erk-2 par l'angiotensine indiquant que la voie décrite pour le récepteur $\beta 2$-adrénergique ne peut être généralisée à tous les RCPG et que d'autres types d'échafaudages existent sans doute.

Si elles sont confirmées, les observations faites par les groupes de Caron et Lefkowitz vont provoquer un changement important dans notre façon d'appréhender la transmission des signaux via les RCPG. Tout d'abord, leurs résultats suggèrent que les protéines $\mathrm{G}$ ne sont plus les seuls partenaires de ces récepteurs pouvant mener à l'activation de voies effectrices. Ils établissent, en outre, que les processus que l'on croyait exclusivement responsables de l'inactivation des voies de signalisation permettent, en fait, de sélectionner l'une de ces voies plutôt qu'une autre. Finalement, les résultats obtenus démontrent qu'à l'image des récepteurs à activité tyrosine kinase, les RCPG peuvent être au cœur d'échafaudages protéiques dont l'organisation modulaire peut être réglée dans le temps selon le niveau d'activation.

M.B.

S.A.

1. Luttrell LM, Ferguson SS, Daaka Y, et al. Betaarrestin-dependent formation of beta2 adrenergic receptor-Src protein kinase complexes. Science 1999; 283: 655-61.

2. Bouvier M, Nantel F, Valiquette M, Moffett S, Mouillac B. Le récepteur $\beta_{2}$-adrénergique. Un modèle d'étude des mécanismes moléculaires de la désensibilisation. Med Sci 1995; 11 : 819-27.

3. Benovic JL, Kuhn H, Weyand I, Codina J, Caron MG, Lefkowitz RJ. Functional desensitization of the isolated beta-adrenergic receptor by the beta-adrenergic receptor kinase: potential role of an analog of the retinal protein arrestin (48-kDa protein). Proc Natl Acad Sci USA 1987; 84: 8879-82.

4. Goodman OB Jr, Krupnick JG, Santini F, et al. Beta-arrestin acts as a clathrin adaptor in endocytosis of the beta2-adrenergic receptor. Nature 1996; 383: 447-50

5. Laporte SA, Oakley RH, Zhang J, et al. The beta-2-adrenergic receptor/beta arrestin complex recruits the clathrin adaptor AP-2 during endocytosis. Proc Natl Acad Sci USA 1999; 96: 3712-7.
6. Chardin P. Protéines Ras et transmission des signaux mitogènes. Med Sci 1994; 10 : 657-64.

7. Luttrell LM, Della Rocca GJ, van Biesen T, Luttrell DK, Lefkowitz RJ. G betagamma subunits mediate Src-dependent phosphorylation of the epidermal growth factor receptor. A scaffold for $\mathrm{G}$ protein-coupled receptor-mediated Ras activation. J Biol Chem 1997; 272: 4637-44.

8. Daaka Y, Luttrell LM, Ahn S, et al. Essential role for $\mathrm{G}$ protein-coupled receptor endocytosis in the activation of mitogen-activated protein kinase. $J$ Biol Chem 1998; 273: 685-8.

9. Vieira AV, Lamaze C, Schmid S. Control of EGF receptor signaling by clathrin-mediated endocytosis. Science 1996; 274: 2086-9.

10. van Biesen T, Hawes BE, Luttrell DK, et al. Receptor-tyrosine-kinase- and $G$ beta gammamediated MAP kinase activation by a common signalling pathway. Nature 1995; 376: 781-4.

11. Pitcher JA, Inglese J, Higgins JB, et al. Role of beta gamma subunits of $G$ proteins in targeting the beta-adrenergic receptor kinase to membrane-bound receptors. Science 1992; 257: 1264-7.

12. Chardin P. Rôle du domaine PH dans le recrutement membranaire des facteurs d'échange pour les petites protéines G. Med Sci 1997; 13: 731-4. 13. Gerhardt CC, Gros J, Strosberg AD, Issad T. Stimulation of the extracellular signal-regulated kinase $1 / 2$ pathway by human beta-3 adrenergic receptor: new pharmacological profile and mechanism of activation. Mol Pharmacol 1999; 55 : 255-62.

14. Ishida M, Ishida T, Thomas SM, Berk BC. Activation of extracellular signal-regulated kinases (ERK1/2) by angiotensin II is dependent on c-Src in vascular smooth muscle cells. Circ Res 1998; $82: 7-12$

15. Liggett SB, Freedman NJ, Schwinn DA, Lefkowitz RJ. Structural basis for receptor subtype-specific regulation revealed by a chimeric beta 3/beta 2-adrenergic receptor. Proc Natl Acad Sci USA 1993; 90 : 3665-9.

16. Nantel F, Bonin H, Emorine LJ, Zilberfarb V, Strosberg AD, Bouvier M, Marullo S. The human beta 3-adrenergic receptor is resistant to short term agonist-promoted desensitization. Mol Pharmacol 1993; 43: 548-55.

17. Zhang J, Ferguson SSG, Barak LS, Menard L, Caron MG. Dynamin and beta-arrestin reveal distinct mechanisms for $\mathrm{G}$ protein-coupled receptor internalization. J Biol Chem 1996; 271 : 18302-5.

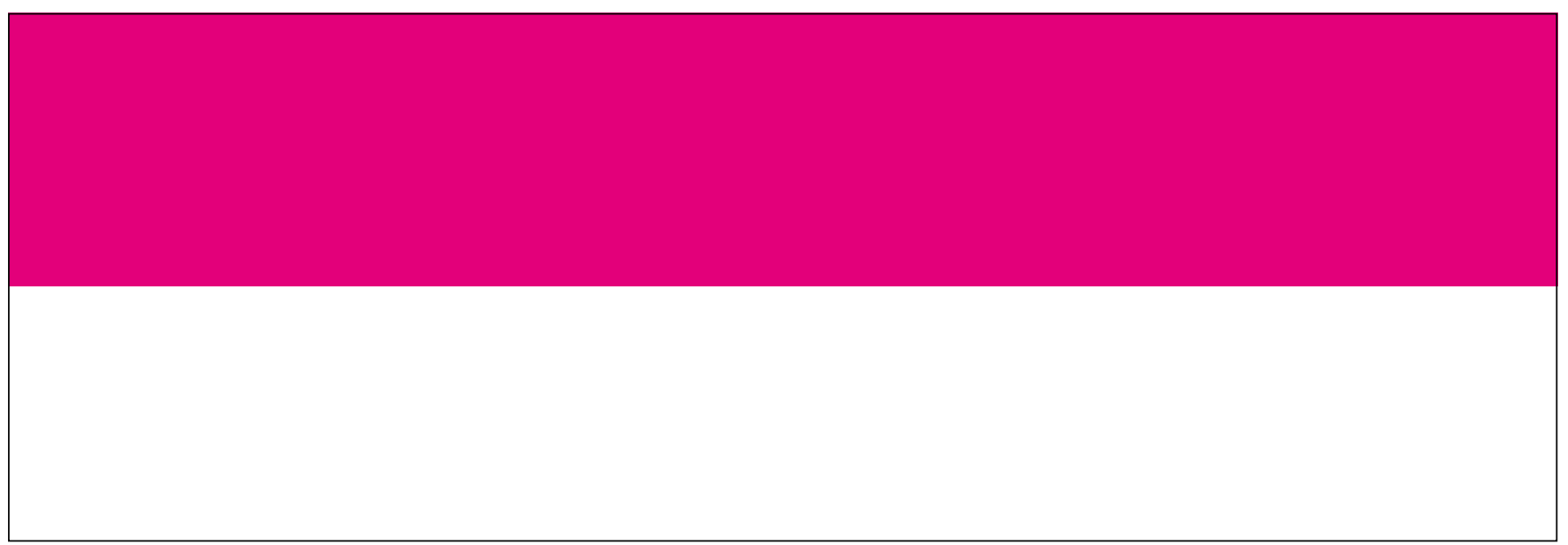


Immunosuppression par le virus de l'hépatite $\mathbf{C}$. Chez une proportion importante de patients infectés par le VHC, la maladie initiale progresse vers la chronicité. Cette forte incidence suggère que le virus est capable d'échapper à la réponse de l'hôte probablement en inhibant ou en modifiant la réponse immunitaire durant la phase aiguë de l'infection. L'étude d'un tel mécanisme d'échappement est rendue difficile parce que le $\mathrm{VHC}$ n'infecte que l'homme et le chimpanzé et qu'il n'existe pas de système de réplication virale in vitro. Des virus de la vaccine recombinés ont été construits pour contourner ce pro- blème. Les effets immunomodulateurs des protéines du VHC ont ainsi pu être testés en comparant la réponse des souris à l'infection par les virus modifiés de la vaccine et par le virus sauvage [1]. L'infection par le virus sauvage a été bien étudiée et est contrôlée par la réponse cytotoxique. Certains virus recombinants exprimant les protéines structurales du VHC provoquent des infections létales, et inhibent la réponse cytotoxique antivirale et la production de cytokines proinflammatoires. La core protéine du VHC est responsable de cet effet immunosuppresseur. Les auteurs font l'hypothèse d'une action directe de cette protéine au niveau des lymphocytes cytotoxiques anti-VHC passant par l'inhibition de leur activation en effecteurs producteurs d'interféron $\gamma$. Il est vrai que l'interaction connue de cette protéine avec le récepteur de la lymphotoxine $\beta$ pourrait interférer à la fois avec le développement de la réponse naturelle précoce et la production de cellules cytotoxiques spécifiques du virus $[2,3]$.

[1. Large MK, et al. J Immunol 1999; 162: 931-8.]

[2. Matsumoto M, et al. J Virol 1997;

71: 1301-9.]

[3. Chen C, et al. I Virol 1997; 71 : 9417-26.] 